\section{Evolução e determinantes de incremento de peso (por altura e idade) em crianças de dois a 72 meses em Sorocaba, São Paulo: evidências a partir de estudo quase- experimental seriado.}

\section{Evolution and determinants of weight gain (weight-for-height and weight-for-age) in children between two and 72 months old in Sorocaba, São Paulo: evidences from a serial quasi-experimental study.}

Neusa Nunes da Silva e Gonçalves 1

Ana Maria Segall Corrêa 2

Carlos Roberto Padovani 3

Liciana Vaz Arruda Silveira Chalita 4

Aguinaldo Gonçalves 5

Gabriela Picarelli Russo Leite 6

1,2,6 Departamento de Medicina Preventiva e Social. Faculdade de Ciências Médicas da Universidade Estadual de Campinas, São Paulo.

R. Luverci Pereira de Souza, 1151, Campinas, SP, CEP: 13.084031

3,4 Departamento de Bioestatística, Instituto de Biociências, Universidade Estadual Paulista, Campus de Botucatu, São Paulo. 5 Grupo de Saúde Coletiva e Atividade Física, Faculdade de Educação Física, Universidade Estadual de Campinas, São Paulo.

\begin{abstract}
Objectives: to review the main aspects of a project conducted by the authors during the last decade with the aim of presenting elements of nutritional supplement in childhood in relation to public policies.

Methods: the growth and development indicators had been accompanied every three months during one year in a population of two to 72 month old children in the outskirts of Sorocaba, São Paulo, Brazil. After completing a child census in two regions of the city, children who had received nutrional assistance (in number of 164) were compared to 280 who had not. A quantitative analysis was performed by Kappa's and Goodman's tests, and by multivariate models. Bias and confounding were investigated and controlled.

Results: the main information obtained refers to the characteristics of the assisted group: 1) higher average age; 2) lower rates of natural milk feeding; 3) lower values of weight/height at admission and greater gains during evolution; 4) this improvement grew with the length of exposure and was greater in the oldest children.

Conclusions: emphasize that today in our country assistance to preschool children should be a mandatory social policy.

Key words Height Weight, Supplementary feeding,
\end{abstract} Nutrition policy, Infant nutrition.

\section{Resumo}

Objetivos: recupera-se síntese dos principais aspectos das publicações referentes a projeto conduzido pela equipe, na década passada, com o objetivo de subsidiar a avaliação de política pública setorial de suplementação alimentar na infância.

Métodos: acompanharam-se trimestralmente, $d u$ rante um ano, indicadores de crescimento e desenvolvimento em populações periféricas de dois a 72 meses de idade, em Sorocaba, São Paulo. Após realização do censo infantil de duas regiões da cidade, as crianças freqüentes a creches (em número de 164) foram comparadas às demais (280). O impacto nutricional foi quantificado através de análises univariadas (testes inferenciais Kappa e de Goodman) e multivariadas. Vieses e confundimento foram averiguados e controlados.

Resultados: observou-se, entre muitas outras informações obtidas, que o grupo assistido apresentou: 1) idade média superior; 2) menor taxa de aleitamento natural; 3) menores valores de peso/altura à admissão, com maiores ganhos durante a evolução; 4) essa melhora aumentou com a duração da exposição e foi maior entre os mais velhos.

Conclusões: avalia-se que assistir apropriadamente pré-escolares constitui hoje, em nosso meio, relevante imperativo de ordem social.

Palavras-chave Peso-Estatura, Suplementação alimentar, Política de nutrição, Nutrição infantil. 
Um dos cortes de aplicação mais interessantes que o Grupo de Saúde Coletiva e Atividade Física da Universidade Estadual de Campinas (UNICAMP), São Paulo, tem efetivado refere-se a estudo em que se procedeu a observação trimestral, durante um ano, de quantificadores de crescimento e desenvolvimento de populações periféricas de dois a 72 meses de idade, em Sorocaba, São Paulo. Tratou-se de um estudo amplo, executado a pedido de autoridades municipais, com a finalidade de subsidiar a adoção de política pública setorial referente a suplementação alimentar para essa faixa etária. Foram identificadas duas regiões da cidade cujas creches estavam iniciando suas atividades e, após proceder-se a censo local das crianças (que identificou 444 na região), estas foram comparadas entre si, segundo freqüentassem (164), ou não (280), tais instituições. A presente comunicação formula resenha constituída de cada uma das principais informações geradas pelo projeto e que se encontravam, até então, atomizadas em diferentes publicações, agora recuperadas.

Já numa avaliação dos primeiros resultados obtidos, 1 foi possível registrar que, embora a relação viesse a se inverter com o passar do período, crianças com menores valores de peso/altura eram as que se encontravam aí atendidas. A comunicação imediatamente posterior ${ }^{2}$ revelou, como elemento adicional a essa relação sua face cruel: eram elas também que apresentavam menor freqüência de aleitamento natural. Foram, em decorrência, propostas medidas de incentivo, como treinamento de funcionários, montagem de ambulatório de amamentação, divulgação do valor nutritivo do leite materno entre as mães e trabalho educativo entre as próprias crianças das creches que são, elas mesmas, poderosos veículos de informação.

A adoção de diferentes procedimentos (evolução dos indicadores antropométricos em valores de $z$ score e do perfil nutricional, tanto através da análise de concordância, pelos testes inferenciais Kappa e de Goodman, quanto pela evolução das médias de peso/altura), explicitados em apresentação a outro encontro científico, ${ }^{3}$ permitiu identificar e quantificar o impacto da suplementação alimentar considerada. Tratando-se de explorar os aspectos metodológicos envolvidos, tanto sua natureza quaseexperimental ${ }^{4}$ quanto o controle de vieses e de fatores de confundimento, 5 foram subseqüentemente aprofundados. Neste sentido, pode-se detectar nos resultados obtidos, entre outros aspectos, a interferência do critério de serem admitidas às creches apenas filhos de trabalhadoras registradas. Quanto a eventual confusão, identificou-se a influência da intensidade biológica diferencial do crescimento du- rante as sucessivas fases que constituem a infância.

Abordando-se determinantes, a regressão logísti$\mathrm{ca}^{6}$ ajustou as variações verificadas, considerou as variáveis antecedentes e de intercorrências à Saúde e demonstrou o efeito positivo da assistência prestada aos pré-escolares, isolando-se destes resultados a influência de outros fatores, como a prevalência de internação hospitalar anterior (maior no grupo assistido). Complementarmente, contextualizou-se que a atividade física pode se constituir em mais um fator a trazer conseqüências favoráveis ao crescimento e desenvolvimento do grupo considerado, quando se comparam grupos ativos e sedentários. ${ }^{7}$

Aferindo simultaneamente a significância nas comparações das diferenças, tanto entre observações sucessivas para o mesmo estrato, quanto na distribuição entre os distintos grupos de alocação (através do teste de Goodman para variações entre e dentro de proporções multinomiais), constatou-se que a melhora havida no grupo de intervenção aumentou com a duração e foi maior entre crianças mais velhas, 8 permitindo concluir que a assistência à creche melhorou-lhes expressivamente a nutrição.

Em síntese, 9 demonstrou-se o impacto nutricional nas condições estudadas, reiterando uma vez mais que assistir apropriadamente crianças em idade escolar constitui hoje, no Brasil, imperativo de ordem social: tanto por razões ligadas à crescente proporção de mulheres que buscam o mercado de trabalho, quanto pela obrigação governamental, em todos os níveis. Trata-se de prover políticas compensatórias dirigidas a segmentos populacionais que vêem oportunidades de emprego e salário diminuirem em virtude das opções do ajuste econômico hoje vigentes.

\section{Agradecimentos}

Fundo de Apoio ao Ensino e Pesquisa da Universidade Estadual de Campinas, (UNICAMP), São Paulo, Prefeitura Municipal de Sorocaba e Conselho Nacionl de Desenvolvimento Científico e Tecnológico. 


\section{Referências}

1. Russo GP, Gonçalves NNS, Corrêa AMS, Gonçalves A, Padovani, CR. Análise de impacto nutricional de programas de alimentação em creches do município de Soroca-

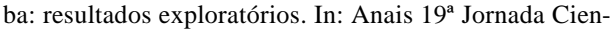
tífica Adunesp; 1995 out 15-18; Botucatu, São Paulo. Botucatu, São Paulo: Associação dos Docentes da Universidade Estadual Paulista (ADUNESP); 1995. p. 23.

2. Russo GP, Gonçalves NNS, Corrêa AMS, Gonçalves A, Padovani CR. Desmame precoce: a face perversa das creches: estudo de Vila Sabiá, Sorocaba, SP. In: Congresso do Cone Sul de Aleitamento Materno; 1996 out 2630; Joinville, Santa Catarina.

3. Gonçalves A, Corrêa AMS, Gonçalves NNS, Leite GPR, Padovani CR. Análise do programa de alimentação em creches do município de Sorocaba: estudo quase-experimental de impacto nutricional. In: Anais Congresso de Educação, Cultura e Ecologia; 1996; Sorocaba, São Paulo. Sorocaba, São Paulo; 1996. p.111

4. Gonçalves A, Corrêa AMS, Padovani CR, Gonçalves NNS, Leite GPR. Ensaios quase-experimentais em avaliação de saúde: contribuições a partir de projeto sobre impacto nutricional em Sorocaba, SP. In: Anais V Congresso Brasileiro Saúde Coletiva; V Congresso Paulista de Saúde Pública; 1997, ago 25-29; Águas de Lindóia, São Paulo. São Paulo: Associação Paulista de Saúde Pública; 1997. p. 273.

5. Gonçalves NNS, Corrêa AMS, Leite GPR, Padovani CR, Gonçalves A. Estudo quase-experimental de impacto nutricional do programa de alimentação em creches do município de Sorocaba, SP: plano metodológico e controle de vieses e confundimento. In: Anais $49^{\mathrm{a}}$ Reunião Anual da Sociedade Brasileira para o Progresso da Ciência, SBPC; 1997 jul 13-18; Belo Horizonte, Minas Gerais. São Paulo: SBPC; 1997. p. 123
6. Corrêa AMS, Leite GPR, Padovani CR, Gonçalves NNS, Gonçalves A. Evolução do estado nutricional de crianças de 3 a 72 meses de idade assistidas em creche, Sorocaba, SP: abordagem de determinantes. In: Anais V Congresso Brasileiro Saúde Coletiva; V Congresso Paulista Saúde Pública; 1997 ago 25-29; Águas de Lindóia, São Paulo. São Paulo: Associação Paulista de Saúde Pública; 1997. p.107-8

7. Gonçalves A, Corrêa AMS, Gonçalves NNS, Leite GPR, Padovani CR. Nutrição para aptidão física: estudo quaseexperimental de pré-escolares na cidade de Sorocaba, SP. In: $1^{\circ}$ Simpósio Fitness Brasil Ciências do Movimento; 1997 abr 30 - maio 2; Santos, São Paulo. São Paulo: Mecânica; 1997.

8. Corrêa AMS, Gonçalves NNS, Gonçalves A, Leite GPR, Padovani CR. Evolução da relação entre peso e altura e idade em crianças de 3 meses a 6 anos assistidos em creches, Sorocaba, SP. Rev Panam Salud Publica 1999; 6: 26-33.

9. Gonçalves A, Chalita LVS, Corrêa AMS, Gonçalves NNS, Padovani CR, Leite GPR. Determinantes da evolução do peso/altura e peso/idade de crianças de 02 meses a 6 anos assistidas em creches, Sorocaba, SP: análise por modelo multivariado em ensaio quase-experimental. In: Livro de Resumos $6^{\circ}$ Congresso Brasileiro de Saúde Coletiva; 2000 ago 28 - set 1; Salvador, Bahia. [Apresentado em: Cienc Saúde Colet 2000; Suppl. 5: 535. 